\title{
Um Modelo para Assistência Educacional Ubíqua orientado a Doenças Crônicas Não Transmissíveis
}

\author{
Andrêsa Vargas Larentis ${ }^{1}$, Débora Nice Ferrari Barbosa ${ }^{2}$, Carla Rosana da Silva ${ }^{3}$, \\ Jorge Luis Victória Barbosa ${ }^{1}$ \\ ${ }^{1}$ Programa de Pós-Graduação em Computação Aplicada (PPGCA) \\ Universidade do Vale do Rio dos Sinos (UNISINOS) \\ ${ }^{2}$ Programa de Pós-Graduação em Diversidade Cultural e Inclusão Social \\ Universidade Feevale \\ ${ }^{3}$ Associação de Assistência em Oncopediatria (AMO Criança) \\ andresa.vargasegmail.com, deboranice@feevale.br, \\ projetoseamocrianca.com.br, jbarbosa@unisinos.br
}

\begin{abstract}
The ubiquitous learning can contribute to prevention of noncommunicable chronic diseases (NCDs) through a contextualized and personalized education oriented to control of risk factors. This article presents SALUS, a computational model for ubiquitous educational assistance oriented to NCDS. SALUS applies dynamic profiles, analysis of similarity and pattern analysis of contexts histories. An evaluation was conducted through a simulated environment using MIMIC III dataset for the creation of groups of individuals and content recommendation. Five groups were created in each of the three criteria used. At least $65 \%$ of the individuals have similarity between the analyzed variables.
\end{abstract}

Resumo. A educação ubíqua pode contribuir para a prevenção das doenças crônicas não transmissíveis (DCNTs) através de uma assistência educacional contextualizada e personalizada para controle dos fatores de risco. Este artigo apresenta o SALUS, um modelo computacional para assistência educacional ubíqua orientado a DCNTs. SALUS utiliza perfis dinâmicos, análise de similaridade e análise de padrões de históricos de contextos. Uma avaliação foi conduzida através de um ambiente simulado usando dados do MIMIC III para a formação de grupos e recomendação de conteúdos. Cinco grupos foram formados em cada um dos três critérios utilizados. Ao menos 65\% dos indivíduos possuem similaridade entre as variáveis analisadas.

\section{Introdução}

Dados da World Health Organization (WHO) estimam que $71 \%$ das mortes que ocorreram em 2016 foram devido a doenças crônicas não transmissíveis (DCNTs). O tabagismo, o uso nocivo do álcool, uma dieta pouco saudável e a falta de atividade física são os principais fatores de risco das DCNTs [WHO 2018]. Desta forma, ações de promoção da saúde e prevenção de DCNTs devem ser propostas para reduzir o nível de exposição dos indivíduos aos fatores de risco e, ao mesmo tempo, fortalecer sua capacidade de seguir padrões de estilo de vida que favoreçam a boa saúde [WHO 2013]. 
VIII Congresso Brasileiro de Informática na Educação (CBIE 2019)

Anais do XXX Simpósio Brasileiro de Informática na Educação (SBIE 2019)

As tecnologias de informação e comunicação (TICs) têm um papel importante no desenvolvimento de ações que oportunizam o autocuidado e autogestão das condições de saúde. Serviços e informações de saúde podem ser prestados e aprimorados por meio das TICs e aplicados no cuidado à saúde dos indivíduos e à saúde pública. Eletronic Health (e-Health), Ubiquitous Health (u-Health), Telehealth e Mobile Health (m-Health) são termos comumente utilizados para traduzir o uso das TICs no contexto da saúde. Estas tecnologias podem ser utilizadas para ofertar uma variedade de serviços incluindo educação em saúde, comunicação e treinamento e, apoio ao diagnóstico e decisão de tratamento [Kim e Xie 2017].

No que se refere as possibilidades de práticas de ensino e aprendizagem, a educação ubíqua [Cárdenas-Robledo e Peña-Ayala 2018] surge da combinação de diferentes tecnologias com o objetivo de possibilitar atividades de aprendizagem onipresentes, levando em consideração o contexto do indivíduo [Dey 2001]. No contexto da saúde, a educação ubíqua pode ser usada para prevenção de doenças e no acompanhamento após um diagnóstico. Além disso, pode ajudar os indivíduos a compreender, avaliar e usar informações de saúde para fazer julgamentos e tomar decisões na vida cotidiana sobre suas condições de saúde.

Diante deste cenário, um estudo realizado por Larentis et al. (2019) identificou oportunidades de pesquisa relacionadas à ausência de sistemas computacionais baseados em computação ubíqua focados na assistência educacional personalizada em DCNTs. Para atender este tópico de pesquisa emergente e estratégico, este artigo propõe um modelo computacional para assistência educacional ubíqua em DCNTs, denominado SALUS. O modelo utiliza contextos [Dey 2001], históricos de contextos [Wiedemann et al. 2016] e perfis dinâmicos [Wagner et al. 2014] na assistência educacional personalizada e adaptada as necessidades do indivíduo. O SALUS possibilita a implementação de serviços inteligentes, que podem ser utilizados por exemplo na predição [Rosa et al. 2016] de DCNTs através da análise de similaridade [Wiedemann et al. 2016] e padrões [Dupont et al. 2019] de históricos de contextos.

Este artigo está estruturado da seguinte forma. A seção 2 apresenta os trabalhos relacionados. A seção 3 propõe o SALUS. Por sua vez, a seção 4 descreve uma avaliação focada em um serviço inteligente para formação de grupos similares para auxiliar no aprendizado das DCNTs. Finalmente, a seção 5 aborda as conclusões finais.

\section{Análise de Trabalhos Relacionados}

O estudo apresentado por Larentis et al. (2019) identificou trabalhos que se relacionam com o SALUS. Eles focam em propostas que contemplam a promoção da educação em DCNTs e foram analisados nos seguintes aspectos: suporte à autogestão e autocuidado, auxílio na educação sobre a doença, envio de mensagens e lembretes, uso de fontes externas, suporte ao conteúdo personalizado, sensibilidade ao contexto e doença crônica.

Abaza e Marschollek (2017) propuseram o uso de um serviço de short message service (SMS) para o envio de mensagens para pacientes diabéticos. O conteúdo das mensagens foi extraído de uma publicação da WHO Eastern Mediterranean Region sobre educação em diabetes e dos padrões do Egyptian Ministry of Health sobre educação do paciente. Alotaibi (2015) propôs um programa educacional para pacientes com diabetes em jejum. Um aplicativo foi utilizado para enviar SMS aos pacientes. As 
VIII Congresso Brasileiro de Informática na Educação (CBIE 2019)

Anais do XXX Simpósio Brasileiro de Informática na Educação (SBIE 2019)

mensagens foram preparadas por uma enfermeira e o conteúdo baseou-se nas diretrizes do National Institute for Health and Care Excellence (NICE). Guo et al. (2015) desenvolveram um aplicativo para pessoas com diabetes para três dimensões: aquisição de conhecimento, mudança de comportamento e eficácia no autocuidado. O conteúdo foi baseado nas diretrizes da American Association of Diabetes Education (AADE) e da Taiwan Association of Diabetes Educators. Hidalgo et al. (2014) propõem a aplicação glUCModel para autogestão de diabetes. A aplicação propõe a melhora da comunicação e interação entre pacientes e médicos. Um sistema de recomendação utiliza soluções adotadas por estudos de casos, encontra semelhanças e cria recomendações ao paciente para melhora dos hábitos e conhecimentos sobre a doença. Mendes Neto et al. (2014) propuseram um modelo que fornece conteúdo personalizado para assistência educacional em pacientes com doenças crônicas. O modelo utiliza dados do perfil, ontologias flexibilizam a integração de serviços e um algoritmo genético suporta a recomendação de conteúdos. Partridge et al. (2017) apresentaram uma plataforma web que permite acesso a conteúdo para pessoas com diabetes tipo 1 . O conteúdo educacional segue o currículo formal do Beta Cell Education Resources for Training in Insulin and Eating (BERTIE). Recursos, tais como vídeos, clips do CYouTube, questionários e desafios, são utilizados para envolver as pessoas em cenários da vida real sobre condições de diabetes. A fim de comparar os trabalhos relacionados, a Tabela 1 apresenta os critérios de comparação e o SALUS. A contribuição deste trabalho está na assistência educacional utilizando sensibilidade ao contexto para educar o indivíduo por toda a vida, no âmbito da prevenção e acompanhamento de DCNTs.

Tabela 1. Comparação dos trabalhos relacionados.

\begin{tabular}{|c|c|c|c|c|c|c|c|}
\hline $\begin{array}{l}\text { Autores/Critérios de } \\
\text { comparação }\end{array}$ & $\begin{array}{l}\text { Autogestão/ } \\
\text { autocuidado }\end{array}$ & $\begin{array}{l}\text { Educação } \\
\text { em DCNTs }\end{array}$ & $\begin{array}{c}\text { Mensagens/ } \\
\text { lembretes }\end{array}$ & Fonte de informação & $\begin{array}{c}\text { Conteúdo } \\
\text { personalizado }\end{array}$ & $\begin{array}{l}\text { Sensível } \\
\text { contexto }\end{array}$ & Doença crônica \\
\hline Abaza e Marschollek (2017) & Sim & Sim & Sim & Provedor de conteúdo & Não & Não & Diabetes \\
\hline Alotaibi (2015) & Sim & Sim & Sim & Provedor de conteúdo & Não & Não & Diabetes tipo 2 \\
\hline Guo et al. (2015) & Sim & Sim & Sim & Provedor de conteúdo & Não & Não & Diabetes \\
\hline Hidalgo et al. (2014) & Sim & Sim & Sim & Estudos de caso & Sim & Não & Diabetes \\
\hline Mendes Neto et al. (2014) & Não & Sim & Não & Sites na internet & Sim & Não & Doenças crônicas \\
\hline Partridge et al. (2017) & Não & Sim & Não & Provedor de conteúdo & Não & Não & Diabetes tipo 1 \\
\hline SALUS & Sim & Sim & Sim & $\begin{array}{l}\text { Provedor de conteúdo } \\
\text { / Sites na internet }\end{array}$ & Sim & Sim & DCNTs \\
\hline
\end{tabular}

\section{SALUS}

O SALUS propõe a criação de um modelo computacional para assistência educacional ubíqua orientado a DCNTs baseado em serviços inteligentes. Para isso, aplica análise de similaridade [Wiedemann et al. 2016] e análise de padrões [Dupont et al. 2019] de históricos de contextos e perfis dinâmicos [Wagner et al. 2014]. O SALUS concentra-se em atender as necessidades do indivíduo de acesso à informação sobre DCNTs para a manutenção e melhoria da sua saúde O modelo proporciona autonomia e independência ao indivíduo para uma aprendizagem mais significativa de modo transparente, empregando para isto técnicas que consideram dados históricos do indivíduo.

Analisando-se os trabalhos relacionados, observou-se que estes referem-se a sistemas que fornecem suporte educacional para pacientes através do uso das TICs. Os sistemas educam pacientes com DCNTs utilizando recursos, tais como SMS, curso elearning e aplicações web e móveis. Entretanto, nenhuma das propostas abordou uma aprendizagem personalizada e contextualizada baseada em históricos de contextos para a criação de serviços educacionais que suportem a promoção da educação em saúde para a prevenção e acompanhamento destas doenças. 
VIII Congresso Brasileiro de Informática na Educação (CBIE 2019)

Anais do XXX Simpósio Brasileiro de Informática na Educação (SBIE 2019)

O SALUS possui os seguintes princípios: 1) educar o indivíduo por toda a vida, e para isso aplica análise de similaridade e análise de padrões de históricos de contextos, considerando dados pessoais e clínicos do indivíduo, para promover uma aprendizagem mais significativa direcionada aos seus interesses; e 2) melhorar a qualidade de vida e saúde do indivíduo. O SALUS permite a criação de serviços educacionais, tais como a formação de grupos por similaridade para a recomendação de conteúdos educacionais em saúde e notificações contendo alertas e lembretes para motivar o indivíduo em atividades de saúde e bem-estar.

\subsection{Visão geral}

A Figura 1 apresenta uma visão geral dos elementos que fazem parte do SALUS: a) Interface do Indivíduo; b) Interface Administrativa; c) Módulo Contexto; d) Módulo Perfil; e) Módulo Serviços; f) Base de Dados Externas; g) Agentes; e h) Banco de Dados. A Interface do Indivíduo permite a interação com as funcionalidades do SALUS de assistência educacional em DCNTs. Uma interface intuitiva é disponibilizada para o indivíduo acessar os serviços disponíveis, tais como avaliação dos conhecimentos sobre a doença, notificações sobre medicamentos e prática de esportes e predições de risco para desenvolver uma doença. A Interface Administrativa possibilita criar e manter configurações. Através de uma interface web, pode-se realizar inserção, atualização e deleção dos dados.

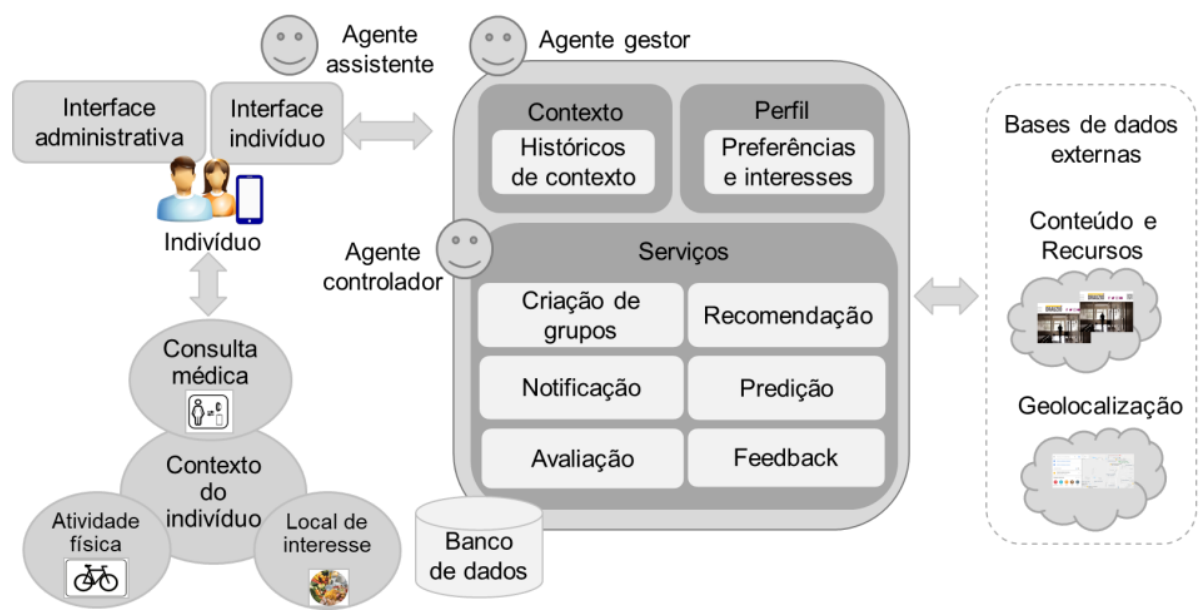

Figura 1. Visão geral do SALUS.

O Módulo Contexto gerencia as informações de contexto onde os indivíduos estão inseridos. Os dados são registrados em tabelas do banco de dados de forma sequencial. $\mathrm{O}$ último registro armazenado representa o contexto mais recente. Os históricos de contexto representam a história do indivíduo e estabelecem uma base de dados dos hábitos, tais como caminhos percorridos, atividades realizadas e recursos utilizados, viabilizando análises e correlações futuras. Os dados servem para extração de informações auxiliares no apoio aos serviços oferecidos pelo modelo.

O Módulo Perfil gerencia as informações do indivíduo. Este módulo armazena as entradas de dados do indivíduo, tais como nome, e-mail, idade, sexo, peso, altura, se fumante, se diabético, se hipertenso, glicose, pressão arterial sistólica e diastólica, colesterol total, colesterol HDL, doenças crônicas, medicamentos, médicos, atividades físicas, histórico de saúde de familiares e preferências. O modelo armazena os dados de 
VIII Congresso Brasileiro de Informática na Educação (CBIE 2019)

Anais do XXX Simpósio Brasileiro de Informática na Educação (SBIE 2019)

tal forma que não é possível identificar o indivíduo (anonimização). Cada indivíduo recebe um código exclusivo.

O Modulo Serviços permite a criação de serviços educacionais para promover a prevenção de doenças, a promoção da saúde, o engajamento do indivíduo e sua participação em atividades de autogestão e autocuidado das suas condições de saúde. Alguns exemplos de serviços que podem ser ofertados: a) formação de grupos para educação e controle das DCNTs conforme o grau de similaridade entre os indivíduos; b) recomendação de conteúdo personalizado, recursos e envio de notificações fazendo uso do processamento de informações armazenadas nos históricos de contextos e do perfil; c) predições de doenças futuras para auxiliar o indivíduo na adoção de hábitos saudáveis e no autocuidado das suas condições de saúde; e d) capacitação do indivíduo através de questionários e quizzes, avaliação do conhecimento adquirido e feedback das avaliações. O feedback pode ser fornecido ao indivíduo a cada interação com a aplicação com o objetivo de motivá-lo a melhorar sua condição de saúde.

Base de Dados Externas são utilizadas para fornecer conteúdos e recursos, tais como a Sociedade Brasileira de Diabetes (provedoras de conteúdo) e a localização de uma Unidade Básica de Saúde (provedora de assistência médica ao indivíduo). Os agentes realizam atividades específicas no SALUS. O Agente Assistente é responsável por monitorar o indivíduo e coletar informações sempre que necessário. O Agente Gestor mantém os dados de perfil, contexto e históricos de contextos atualizados. $\mathrm{O}$ Agente Controlador gerencia as solicitações dos serviços disponíveis.

\subsection{Arquitetura}

A arquitetura do SALUS utiliza o modelo TAM [TAM 2007] e é definida como clienteservidor, conforme apresentado na Figura 2. O modelo está organizado em quatro partes: Client, Server, Database e Service Provider. O Client consome a informação contextual gerada e disponibiliza as aplicações para a interação do indivíduo com o Server. O Client pode ser um site web para manutenção de informações utilizadas pelos módulos do SALUS e um aplicativo para uso em dispositivos móveis. Além disso, o Client reside no dispositivo móvel do indivíduo e suporta as aplicações disponíveis: perfil, configurações e serviços. O Agente Assistente tem como objetivo servir de interface entre o indivíduo e a aplicação, auxiliando-o a consumir os serviços disponíveis de acordo com suas preferências, interesses e contexto. As informações de perfil do indivíduo são coletadas através da sua interação com a aplicação.

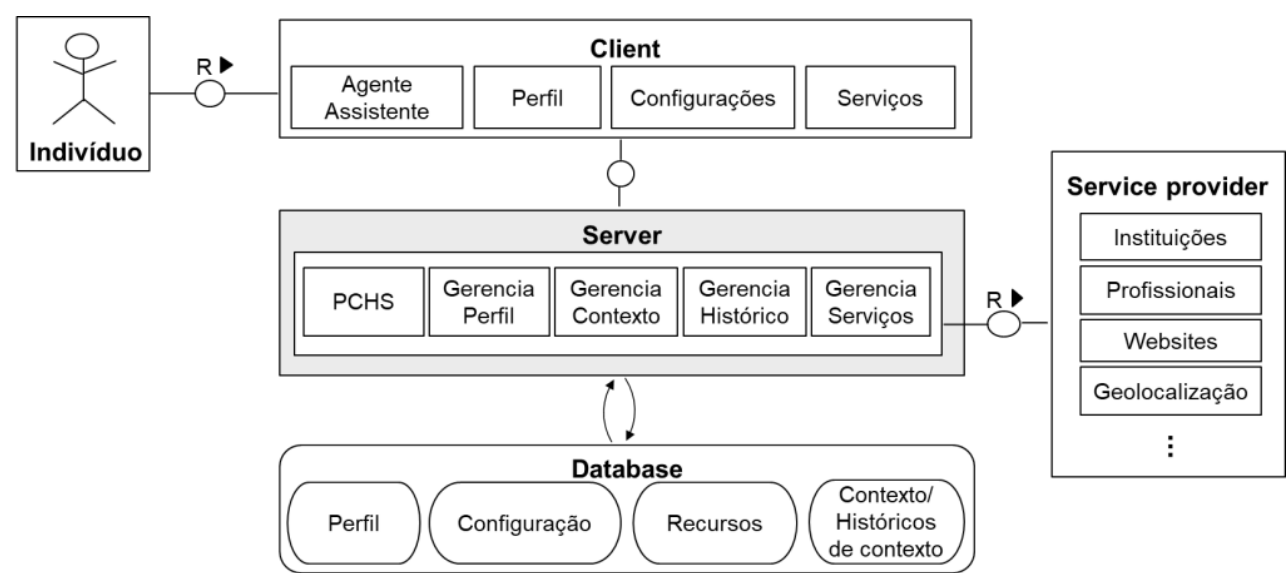

Figura 2. Arquitetura do SALUS. 
VIII Congresso Brasileiro de Informática na Educação (CBIE 2019)

Anais do XXX Simpósio Brasileiro de Informática na Educação (SBIE 2019)

O Server suporta os serviços utilizados pela aplicação para a manutenção do perfil do indivíduo, gerenciamento de contexto e históricos de contextos e gerenciamento de serviços para assistência educacional em saúde. O Server é responsável por adquirir e processar os dados das entidades monitoradas, convertendoos em informação contextual. Além disso, o Server possui na sua estrutura cinco elementos: a) Perfil, Contexto, Histórico de Contextos e Serviços (PCHS); b) Gerencia Perfil; c) Gerencia Contexto; d) Gerencia Histórico; e) Gerencia Serviços. O PCHS é um sistema multiagente [Haeng-Kon 2014] responsável por monitorar as entidades e gerenciar as requisições de serviços disponíveis. O Database armazena os dados do perfil do indivíduo, das configurações da aplicação, dos recursos disponíveis para uso, além de contextos e históricos de contextos visitados pelo indivíduo. O Service Provider é responsável por gerenciar provedores de serviços externos. Mecanismos de buscas são utilizados para localizar fontes de informação na internet relacionadas as DCNTs. Serviços de geolocalização são utilizados para determinar a localização de uma entidade e recursos oferecidos.

\section{Avaliação}

Uma avaliação do SALUS foi conduzida através de uma simulação desenvolvida na linguagem R. Definiu-se como objetivo da simulação a criação de um serviço inteligente para formação de grupos de indivíduos com características similares e recomendação de conteúdo dentro de um contexto hospitalar, considerando dados clínicos e sinais vitais. A simulação utilizou o banco de dados de demonstração do Medical Information Mart for Intensive Care-III (MIMIC-III) [Johnson et al. 2019]. MIMIC-III contém dados de internações de pacientes da unidade de cuidados intensivos do Beth Israel Deaconess Medical Center, localizado em Boston, Massachusetts entre 2001 a 2012 [Goldberger et al. 2000 e Johnson et al. 2016]. A amostra da população foi obtida das tabelas PATIENTS, ADMISSIONS, LABEVENTS, CHARTEVENTS, ITENS e LABITENS, sendo 20 indivíduos selecionados das relações entre as tabelas LABEVENTS x ITENS e CHARTEVENTS x LABITENS através do atributo ITEMID.

A simulação consistiu em: 1) criar um serviço para formação de grupos de indivíduos com características similares e, posterior recomendação de conteúdos educacionais em saúde; 2) normalizar os dados da amostra da população; 3) executar o serviço; e 4) extrair e analisar os resultados. Para a análise de similaridade foi utilizado o conceito de clusterização, ou agrupamento, cujo objetivo é particionar os dados de uma amostra em subconjuntos chamados clusters. De acordo com Goldschmidt e Passos (2005) os elementos que compõem um cluster compartilham características que permitam distingui-los dos demais clusters. O resultado consiste em obter a maior similaridade intra-cluster e a maior distância inter-cluster. Uma matriz de dados é construída a partir da lista de objetos a serem agrupados e uma matriz de similaridade a partir da distância entre pares de objetos. Quanto mais próximo de 0 (zero) for a distância entre dois objetos, mais similares eles serão.

Dentre as medidas de distância, a Euclidiana foi escolhida para a simulação. Como método de formação de agrupamentos foi escolhido o AGglomerative NESting (AGNES) [Kaufman e Rousseeuw 1990]. O AGNES é um algoritmo baseado no método hierárquico aglomerativo, ou seja, cada objeto forma um agrupamento e a cada nova interação os agrupamentos mais próximos são unidos formando um só, de acordo com um critério pré-estabelecido. Entre os critérios de junção pode-se citar o que une os 
VIII Congresso Brasileiro de Informática na Educação (CBIE 2019)

Anais do XXX Simpósio Brasileiro de Informática na Educação (SBIE 2019)

agrupamentos de acordo com a média da dissimilaridade (average linkage) entre os pontos de um agrupamento e outro, o método do vizinho mais próximo (single linkage) que usa a menor distância entre os dois agrupamentos e o método do vizinho mais longe (complete linkage) que usa a maior distância entre os dois agrupamentos. A distância Euclidiana foi incluída como parâmetro da função dist (). A função hclust () executa o algoritmo de clusterização e foi implementada para três critérios de junção: single linkage, complete linkage e average linkage. $\mathrm{O}$ serviço foi executado recebendo como parâmetro o arquivo .csv com os dados normalizados. A Figura 3 apresenta os dendrogramas gerados pelos critérios de junção single linkage e complete linkage. Ambos sugerem a formação de 5 clusters, distintos através das cores preta, vermelha, verde, roxa e azul.

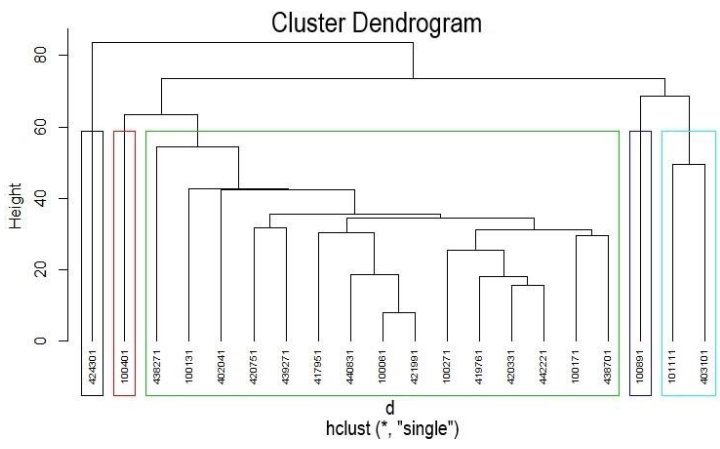

(a)

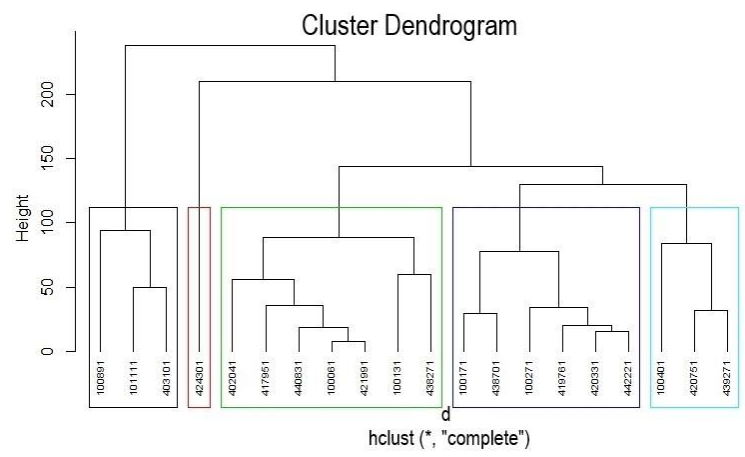

(b)

Figura 3. Dendrograma dos critérios single linkage e complete linkage.

No dendrograma da Figura 3a, o critério single linkage classificou a maior quantidade de indivíduos no grupo 1-Verde (15). Analisando-se o valor das variáveis colesterol HDL, colesterol Total, glicose, pressão arterial sistólica e idade dos indivíduos, observou-se que os indivíduos possuem valores muito próximos da média das variáveis analisadas, ou seja, são similares entre si, justificando o agrupamento formado. O indivíduo do grupo 2-Vermelho apresenta valor elevado no colesterol HDL e o indivíduo do grupo 3-Roxo no colesterol Total. Os dois indivíduos pertencentes ao grupo 4-Azul possuem valores altos do colesterol Total. Por fim, o indivíduo pertencente ao grupo 5-Preto apresentou um valor elevado da glicose. A Figura 3b ilustra o complete linkage, sendo o grupo 1-Verde (7 indivíduos), grupo 2-Roxo (6), grupo 3-Azul (3), grupo 4-Preto (3) e grupo 5-Vermelho (1). O critério average linkage apresentou os resultados: grupo 1-Azul (13 indivíduos), grupo 2-Verde (2), grupo 3Roxo (1), grupo 4-Preto (3) e grupo 5-Vermelho (1).

Posterior a isso, relacionou-se conteúdos para auxiliar na prevenção e acompanhamento de DCNTs dos indivíduos por grupos. Uma avaliação identificou quais palavras-chave se relacionam com cada uma das variáveis avaliadas por exemplo: a) "colesterol Total" e "colesterol HDL" foi relacionado as palavras-chave colesterol alto e hipercolesterolemia; b) "glicose" as palavras-chave glicemia e glicose alta; c) "idade" as palavras-chave geriatria, velhice e terceira idade; e d) "pressão arterial" as palavras-chave hipertensão, hipotensão, pressão alta e pressão baixa. Adicionalmente, foi realizada uma pesquisa para identificar quais os conteúdos de maior relevância para 
VIII Congresso Brasileiro de Informática na Educação (CBIE 2019)

Anais do XXX Simpósio Brasileiro de Informática na Educação (SBIE 2019)

as palavras-chave através da ferramenta SEMrush ${ }^{1}$. Esta ferramenta fornece dados de natureza diversa incluindo consumo de informações, uso de palavras-chave e acesso a sites na internet. $\mathrm{O}$ resultado apresentou referências de 1.179 conteúdos mais acessados quando uma pesquisa é realizada em um motor de busca da internet, como o () Google.

Uma vez mapeados os conteúdos de cada palavra-chave, que por sua vez se relacionam com as variáveis avaliadas, analisou-se que variáveis mais influenciaram nos grupos formados, de forma a indicar para estes quais conteúdos poderiam ser relevantes na prevenção e acompanhamento de DCNTs. A Tabela 2 apresenta a relação do grupo versus variáveis de maior influência e a quantidade de conteúdos associados. A distribuição foi realizada para cada um dos grupos formados pelos critérios utilizados single linkage, complete linkage e average linkage. A relação foi arbitrada através dos resultados obtidos. A partir disso, foram sugeridos conteúdos considerando os dados históricos do período que o indivíduo ficou internado na unidade de cuidados intensivo. No critério single linkage, os grupos 3-Roxo (1) e 5-Preto (1) tiveram uma menor quantidade de conteúdos recomendados (196 e 294, respectivamente) em relação aos grupos 2-Vermelho (1) e 4-Azul (2) (490 e 492, respectivamente) devido a influência das variáveis na similaridade dos indivíduos destes grupos. Já os indivíduos do grupo 1Verde que representam 75\% (15/20), receberam os 1.179 conteúdos. Neste caso, analisando os dados dos indivíduos, não foi identificada uma variável de maior influência, sendo assim a recomendação de conteúdos focou nos aspectos de prevenção e acompanhamento dos cuidados com a saúde. No critério complete linkage, indivíduos dos grupos 1-Verde (7) e 2-Roxo (6), que representam 65\% (13/20), receberam a totalidade dos conteúdos extraídos da pesquisa, ou seja 1.179. No critério average linkage, indivíduos do grupo 1-Azul (13), que representam 65\% (13/20) receberam também os mesmos 1.179 conteúdos.

Tabela 2. Distribuição de conteúdos por critérios $\mathrm{x}$ grupos $\mathrm{x}$ variáveis.

\begin{tabular}{clcclccc}
\hline Grupo & $\begin{array}{c}\text { Single linkage } \\
\text { Variáveis maior } \\
\text { influência }\end{array}$ & Qtde & Grupo & $\begin{array}{c}\text { Complete linkage } \\
\text { Variáveis maior } \\
\text { influência }\end{array}$ & Qtde & Grupo & $\begin{array}{c}\text { Average linkage } \\
\text { Variáveis maior } \\
\text { influência }\end{array}$ \\
\hline 1 & Todas & 1.179 & 1 & Todas & 1.179 & 1 & Todas \\
2 & Colesterol HDL e glicose & 490 & 2 & Todas & 1.179 & 2 & Glicose \\
3 & Colesterol Total & 196 & 3 & Colesterol HDL e glicose & 490 & 3 & Colesterol HDL e glicose \\
4 & Colesterol Total e idade & 492 & 4 & Colesterol Total & 196 & 4 & Colesterol Total \\
5 & Glicose & 294 & 5 & Glicose & 294 & 5 & Glicose \\
\hline
\end{tabular}

\section{Conclusões}

Este artigo propôs o SALUS, um modelo computacional para assistência educacional ubíqua orientado a DCNTs baseado em serviços inteligentes. Para isso, aplica análise de similaridade [Wiedemann et al. 2016] e análise de padrões [Dupont et al. 2019] de históricos de contextos e perfis dinâmicos [Wagner et al. 2014] como forma de suportar uma educação personalizada de acordo com as necessidades do indivíduo no âmbito das DCNTs. Uma simulação foi realizada para avaliação de um serviço inteligente para formação de grupos de indivíduos com características similares e recomendação de conteúdo relacionados as DCNTs. A amostra da população foi obtida do banco de dados MIMIC-III. O resultado apresentou uma proximidade dos grupos formados entre os três critérios de junção utilizados. Cinco grupos de indivíduos foram formados em cada um dos critérios. Observou-se nos grupos formados pelo maior número de indivíduos que a

\footnotetext{
${ }^{1}$ https://www.semrush.com/
} 
VIII Congresso Brasileiro de Informática na Educação (CBIE 2019)

Anais do XXX Simpósio Brasileiro de Informática na Educação (SBIE 2019)

recomendação de conteúdos considerou todas as variáveis avaliadas. Desta forma, 1.179 conteúdos potencialmente úteis aos interesses dos indivíduos foram recomendados. Pode-se indicar que mais de $65 \%$ dos indivíduos inclusos nos grupos formados possuem características similares. Para os $35 \%$ restantes, foram recomendados conteúdos mais específicos, por exemplo colesterol Total (196 conteúdos), colesterol HDL + glicose (490) e somente glicose (294).

Como trabalhos futuros propõe-se uma avaliação para criação de um serviço para predição de DCNTs através da análise de similaridade [Wiedemann et al. 2016] e análise de padrões [Dupont et al. 2019] de históricos de contextos, bem como a possibilidade de avaliação com indivíduos quanto a aceitação dos conteúdos recomendados. Além disso, seria interessante a realização de avaliações para uma melhor mensuração da eficácia dos serviços. Assim, o modelo poderia ser implementado como um módulo experimental em uma aplicação, e então submetido a avaliação por um grupo de indivíduos. Por fim, a identificação de históricos de contextos por análise de similaridade e análise de padrões poderá auxiliar em outras aplicações, como por exemplo, a formação inteligente de grupos de controle de prevenção conforme o grau de similaridade entre os indivíduos, ou ainda a criação de perfis de indivíduos conforme seu histórico de interesses.

\section{Agradecimentos}

Os autores agradecem à FAPERGS, à CAPES - Código de Financiamento 001, ao CNPq, à Universidade do Vale do Rio dos Sinos (Unisinos), à Universidade Feevale e à Associação de Assistência em Oncopediatria (AMO Criança) pelo apoio ao desenvolvimento desse trabalho.

\section{Referências}

Abaza, H., Marschollek, M. (2017) SMS education for the promotion of diabetes selfmanagement in low \& middle income countries: a pilot randomized controlled trial in Egypt. BMC Public Health, 17(962).

Alotaibi, M. (2015) A Mobile Diabetes Educational System for Fasting Type-2 Diabetics in Saudi Arabia. In Proceedings of the 2nd ICITACEE, pages 173-176.

Cárdenas-Robledo, L. A., Peña-Ayala, A. (2018) Ubiquitous learning: A systematic review. Telematics and Informatics, 35(5):1097-1132.

Dey, A. K. (2001) Understanding and Using Context. Journal Personal and Ubiquitous Computing, 5(1):4-7.

Dupont, D., Barbosa, J. L. V., Alves, B. M. (2019) CHSPAM: a multi-domain model for sequential pattern discovery and monitoring in contexts histories. Pattern Analysis and Applications, pages 1-10.

Goldberger, A. L. et al. (2000) PhysioBank, PhysioToolkit, and PhysioNet: Components of a New Research Resource for Complex Physiologic Signals. Circulation, 101(23):215-220.

Goldschmidt, R., Passos, E. (2005) Data mining: um guia prático. RJ: Elsevier.

Guo, S., Chang, H. -K., Lin, C. -Y. (2015) Impact of Mobile Diabetes Self-Care System on patients' knowledge, behavior and efficacy. Computers in Industry, 69:22-29. 
VIII Congresso Brasileiro de Informática na Educação (CBIE 2019)

Anais do XXX Simpósio Brasileiro de Informática na Educação (SBIE 2019)

Haeng-Kon, K. (2014). Convergence agent model for developing u-healthcare systems. Future Generation Computer Systems, 35:39-48.

Hidalgo, J. I., Maqueda, E., Risco-Martín, J. L., Cuesta-Infante, A., Colmenar, J. M., Nobel, J. (2014) glUCModel: A monitoring and modeling system for chronic diseases applied to diabetes. Journal of Biomedical Informatics, 48:183-192.

Johnson, A. E. W. et al. (2016) MIMIC-III, a freely accessible critical care database. Scientific Data, 3(160035).

Johnson, A., Pollard, T., Mark, R. (2019) MIMIC-III Clinical Database Demo. PhysioNet. Disponível em: https://alpha.physionet.org/content/mimiciii-demo/1.4/. Acesso em: 7 de Julho de 2019.

Kaufman, L., Rousseeuw, P.J. (1990) Finding Groups in Data: An Introduction to Cluster Analysis. New Jersey: John Wiley \& Sons, Inc.

Kim, H., Xie, B. (2017) Health Literacy in the eHealth era: A Systematic review of the literature. Patient Education and Counseling, 100:1073-1082.

Larentis, A. V., Barbosa, D. N. F., da Silva, C. R., Barbosa, J. L. V. (2019) Applied Computing to Education on Noncommunicable Chronic Diseases: A Systematic Mapping Study. Telemedicine and e-Health.

Mendes Neto, F. M. et al. (2014) Content's personalized recommendation for implementing ubiquitous learning in health 2.0. IEEE Latin America Transactions, 12(8):1515-1522.

Partridge, H., Shaban, C., Weiss, M. (2017) Innovating structured education for people with type 1 diabetes: Www.Bertieonline.org.UK. Journal of Diabetes Nursing, 21(7): 255-258.

Rosa, J. H., Barbosa, J. L. V., Ribeiro, G. D. (2016) ORACON: An Adaptive Model for Context Prediction. Expert Systems with Applications, 45(1):56-70.

TAM (2007) Standardized Technical Architecture Modeling - Conceptual and Design Level. Disponível em: http://www.fmc-modeling.org/download/fmc-and-tam/SAPTAM_Standard.pdf. Acesso em: 7 de Julho de 2019.

Wagner, A., Barbosa, J. L. V., Barbosa, D. N. F. (2014) A model for profile management applied to ubiquitous learning environments. Expert Systems with Applications, 41(4):2023-2034.

WHO (2013) Global Action Plan for the Prevention and Control of noncommunicable diseases 2013-2020. Disponível em:

http://www.who.int/nmh/events/ncd_action_plan/. Acesso em: 7 de Julho de 2019.

WHO (2018) World Health Statistics 2018: Monitoring Health for the SDGs, sustainable development goals. Disponível em: http://www.who.int/gho/publications/world_health_statistics/2018/. Acesso em: 7 de Julho de 2019.

Wiedemann, T., Barbosa, J. L. V., Rigo, S. J., Barbosa, D. N. F. (2016) RecSim: A Model for Learning Objects Recommendation using Similarity of Sessions. Journal of Universal Computer Science, 22(8):1175-1200. 